\title{
Thermal Vibration of Laminated Magnetostrictive Plates Without Shear Effects
}

\section{Chih-Chiang Hong}

Department of Mechanical Engineering, Hsiuping University of Science and Technology, Taichung, Taiwan ROC

\section{Email address:}

cchong@mail.hust.edu.tw

\section{To cite this article:}

Chih-Chiang Hong. Thermal Vibration of Laminated Magnetostrictive Plates Without Shear Effects. International Journal of Electrical Components and Energy Conversion. Vol. 3, No. 3, 2017, pp. 63-69. doi: 10.11648/j.ijecec.20170303.12

Received: October 18, 2016; Accepted: October 27, 2016; Published: November 28, 2017

\begin{abstract}
The study of laminated magnetostrictive plate without shear deformation under thermal vibration is calculated by using the generalized differential quadrature (GDQ) method. In the thermoelastic stress-strain relations that containing the linear temperature rise and the magnetostrictive coupling terms with velocity feedback control. The dynamic differential equations without shear deformation are normalized and discrete into the dynamic discretized equations with GDQ method. Four edges of rectangular laminated magnetostrictive plate with simply supported boundary conditions are considered. In the moderately thick plate of laminated magnetostrictive plate, the effect of shear deformation should be considered for the computational controlled values of transverse center deflection and dominated normal stress.
\end{abstract}

Keywords: Magnetostrictive Plate, Shear Deformation, Thermal Vibration, GDQ, Velocity Feedback Control

\section{Introduction}

There were several researches in the transverse displacement of vibrations for the laminated magnetostrictive plate. In 2016, Arani and Maraghi [1] made the study of the linear sinusoidal shear deformation plate theories for vibrations of magnetostrictive plate under follower force by using the differential quadrature method (DQM). There are some studied parameters e.g. follower force, velocity feedback gain, aspect ratio and thickness ratio were used to investigate the vibration behavior for the magnetostrictive plate. In 2015, Zhang et al. [2] used the finite element method (FEM) to analyze the nonlinear effect of constitutive model on the vibration of cantilever laminated composite plate with giant magnetostrictive materials (GMM) layers. Studied parameters in embedded placement of GMM layers and control gain were used to investigate the suppression on vibration. There were several researches in the transverse displacement with the effect of shear deformation for the laminated composite plates. In 2016, Sarangan and Singh [3] used the higher-order shear deformation theories (HSDT) to study the free vibration of laminated composite. Some of the HSDT, e.g. algebraic (ADT), exponential (EDT), hyperbolic (HDT), logarithmic (LDT) and trigonometric (TDT) were studied in Navier closed form solution, there are no transverse shear stresses at the top and bottom of the plate surfaces under free vibration. In 2012, Hong [4] studied the thermal vibration of magnetostrictive functionally graded materials (FGM) plate with the YNS first-order shear deformation theories (FSDT) under rapid heating. The transverse shear stresses exist at the top and bottom of the plate surfaces under thermal vibration and control gain. In 2008, Nguyen et al. [5] made the static numerical analyses for the FGM plate with the effect of shear deformation. Terfenol-D magnetostrictive materials have the magneto-electric coupling property under the action of magnetism and mechanism. In 2006, Ramirez et al. [6] presented the Ritz approach to obtain the free vibration solution for magneto-electro-elastic laminates. In 2005, Lee and Reddy [7] used the finite element method to analyze the non-linear response of laminated plate of magnetostrictive material under thermo-mechanical loading. In 2004, Lee et al. [8] obtained the transient vibration values of displacement for the Terfenol-D magnetostrictive material plate included the effect of shear deformation by using the FEM. In 2014, Hong [9] used the GDQ method with the effect of modified shear correction coefficient to make the thermal vibration analyses of FGM plates and mounted magnetostrictive layer. In 2009, Hong [10] used the GDQ method without the effect of shear coefficient to make the thermal transient response analyses of laminated magnetostrictive plates. It is interesting to study thermal 
vibration in the transverse displacement and thermal stress of the laminated magnetostrictive plates without/with the shear deformation effect by using the GDQ method.

\section{Formulation}

\subsection{Displacement Field}

The time dependent of displacements fields without the shear deformation are assumed in the following equation:

$$
\begin{aligned}
& u=u^{0}(x, y, t) \\
& v=v^{0}(x, y, t) \\
& w=w(x, y, t)
\end{aligned}
$$

where $u^{0}$ and $v^{0}$ are tangential displacements, $w$ is transverse displacement of the middle-plane, $t$ is time.

\subsection{GDQ Method}

The GDQ method approximates the derivative of function, for example, the first-order and the second-order derivatives of function $f^{*}(x, y)$ at coordinates $\left(x_{i}, y_{j}\right)$ of grid point $(i, j)$ can be discretized by [10] [11] [12] and rewritten as follows:

$$
\begin{gathered}
\left.\frac{\partial f^{*}}{\partial x}\right|_{i, j} \approx \sum_{l=1}^{N} A_{i, l}^{(1)} f_{l, j}^{*},\left.\frac{\partial f^{*}}{\partial y}\right|_{i, j} \approx \sum_{m=1}^{M} B_{j, m}^{(1)} f_{i, m}^{*}, \\
\left.\frac{\partial^{2} f^{*}}{\partial x^{2}}\right|_{i, j} \approx \sum_{l=1}^{N} A_{i, l}^{(2)} f_{l, j}^{*}, \\
\left.\frac{\partial^{2} f^{*}}{\partial y^{2}}\right|_{i, j} \approx \sum_{m=1}^{M} B_{j, m}^{(2)} f_{i, m}^{*},\left.\frac{\partial^{2} f^{*}}{\partial x \partial y}\right|_{i, j} \approx \sum_{l=1}^{N} A_{i, l}^{(1)} \sum_{m=1}^{M} B_{j, m}^{(1)} f_{l, m}^{*} .
\end{gathered}
$$

where $A_{i, j}^{(m)}$ and $B_{i, j}^{(m)}$ denote the weighting coefficients for the $m^{\text {th }}$-order derivative of the function $f^{*}(x, y)$ with respect to the $x$ and $y$ directions.

\subsection{Thermoelastic Stress-Strain Relations with Magnetostrictive Effect}

We consider a rectangular laminated magnetostrictive plate of the length $\mathrm{a}$ and $\mathrm{b}$ in the $x, y$ direction, respectively, under uniformly distributed loading and thermal effect as described in [10]. There are no shear stresses and shear strains in the laminate without shear effect assumption. The plane stress in a laminated material with magnetostrictive effect for the $k^{\text {th }}$ layer are in the following equations [7]:

$$
\begin{gathered}
\left\{\begin{array}{c}
\sigma_{x} \\
\sigma_{y} \\
\sigma_{x y}
\end{array}\right\}_{(k)}=\left[\begin{array}{lll}
\bar{Q}_{11} & \bar{Q}_{12} & \bar{Q}_{16} \\
\bar{Q}_{12} & \bar{Q}_{22} & \bar{Q}_{26} \\
\bar{Q}_{16} & \bar{Q}_{26} & \bar{Q}_{66}
\end{array}\right]_{(k)}\left\{\begin{array}{c}
\varepsilon_{x}-\alpha_{x} \Delta T \\
\varepsilon_{y}-\alpha_{y} \Delta T \\
\varepsilon_{x y}-\alpha_{x y} \Delta T
\end{array}\right\}_{(k)} \\
-\left[\begin{array}{ccc}
0 & 0 & \tilde{e}_{31} \\
0 & 0 & \tilde{e}_{32} \\
0 & 0 & \tilde{e}_{36}
\end{array}\right]_{(k)}\left\{\begin{array}{c}
0 \\
0 \\
\tilde{H}_{z}
\end{array}\right\}_{(k)}
\end{gathered}
$$

where $\alpha_{x}$ and $\alpha_{y}$ are the coefficients of thermal expansion, $\alpha_{x y}$ is the coefficient of thermal shear. $\bar{Q}_{i j}$ is the so called transformed reduced stiffness can be in terms of the elastic stiffness of materials and can be explained more detail by Whitney [13].

$$
\varepsilon_{x}, \varepsilon_{y}, \varepsilon_{x y} \text { are in-plane strains. } \Delta T=T_{0}(x, y, t)+\frac{z}{h^{*}} T_{1}(x, y, t)
$$

is the temperature difference between the laminate and curing area, $z$ is the coordinate in the thickness direction. $h^{*}$ is the plate total thickness. $\tilde{e}_{i j}$ is the transformed magnetostrictive coupling moduli. $\tilde{H}_{z}$ is the magnetic field intensity, expressed in the following equation. $\tilde{H}_{z}(x, y, t)=k_{c} \tilde{I}(x, y, t) \quad$ with velocity feedback control $\tilde{I}(x, y, t)=c(t) \frac{\partial w}{\partial t}$ where $k_{c}$ is the coil constant, $\tilde{I}(x, y, t)$ is the coil current, $c(t)$ is the control gain.

\subsection{Dynamic Equilibrium Differential Equations}

Without shear deformation effect, the dynamic equilibrium differential equations in terms of displacements included the magnetostrictive loads are expressed in the following matrix forms [10]:

$$
\begin{aligned}
& {\left[\begin{array}{ccccccccc}
A_{11} & 2 A_{16} & A_{66} & A_{16} & A_{12}+A_{66} & A_{26} & 0 & 0 & 0 \\
A_{16} & A_{12}+A_{66} & A_{26} & A_{66} & 2 A_{26} & A_{22} & 0 & 0 & 0 \\
B_{11}+B_{16} & 2 B_{16}+B_{12}+B_{66} & B_{66}+B_{26} & B_{16}+B_{66} & B_{12}+B_{66}+2 B_{26} & B_{26}+B_{22} & 0 & 0 & 0
\end{array}\right]} \\
& \left\{\frac{\partial^{2} u^{0}}{\partial x^{2}} \frac{\partial^{2} u^{0}}{\partial x \partial y} \frac{\partial^{2} u^{0}}{\partial y^{2}} \frac{\partial^{2} v^{0}}{\partial x^{2}} \quad \frac{\partial^{2} v^{0}}{\partial x \partial y} \frac{\partial^{2} v^{0}}{\partial y^{2}} \quad \frac{\partial^{2} w}{\partial x^{2}} \quad \frac{\partial^{2} w}{\partial x \partial y} \quad \frac{\partial^{2} w}{\partial y^{2}}\right\}^{t} \\
& =\left\{\begin{array}{l}
f_{1} \\
f_{2} \\
f_{3}
\end{array}\right\}+\rho\left[\begin{array}{ccc}
1 & 0 & 0 \\
0 & 1 & 0 \\
0 & 0 & -1
\end{array}\right]\left\{\begin{array}{l}
\frac{\partial^{2} u^{0}}{\partial t^{2}} \\
\frac{\partial^{2} v^{0}}{\partial t^{2}} \\
\frac{\partial^{2} w}{\partial t^{2}}
\end{array}\right\}+H\left[\begin{array}{ll}
0 & 0 \\
0 & 0 \\
1 & 1
\end{array}\right]\left\{\begin{array}{c}
\frac{\partial^{2} u^{0}}{\partial t^{2}} \\
\frac{\partial^{2} v^{0}}{\partial t^{2}}
\end{array}\right\}
\end{aligned}
$$


where $f_{1}, f_{2}, f_{3}$ are the expressions of thermal loads $(\bar{N}, \bar{M})$, mechanical loads $\left(p_{1}, p_{2}, q\right)$ and magnetostrictive loads $(\tilde{N}, \tilde{M})$.

$$
\begin{gathered}
f_{1}=\frac{\partial \bar{N}_{x}}{\partial x}+\frac{\partial \bar{N}_{x y}}{\partial y}+p_{1}+\frac{\partial \tilde{N}_{x}}{\partial x}+\frac{\partial \tilde{N}_{x y}}{\partial y} \quad f_{2}=\frac{\partial \bar{N}_{x y}}{\partial x}+\frac{\partial \bar{N}_{y}}{\partial y}+p_{2}+\frac{\partial \tilde{N}_{x y}}{\partial x}+\frac{\partial \tilde{N}_{y}}{\partial y} \\
f_{3}=\frac{\partial \bar{M}_{x}}{\partial x}+2 \frac{\partial \bar{M}_{x y}}{\partial y}+\frac{\partial \bar{M}_{y}}{\partial y}+q+\frac{\partial \tilde{M}_{x}}{\partial x}+2 \frac{\partial \tilde{M}_{x y}}{\partial y}+\frac{\partial \tilde{M}_{y}}{\partial y} \\
\left(\bar{N}_{x}, \bar{M}_{x}\right)=\int_{\frac{-h^{*}}{2}}^{\frac{h^{*}}{2}}\left(\bar{Q}_{11} \alpha_{x}+\bar{Q}_{12} \alpha_{y}+\bar{Q}_{16} \alpha_{x y}\right)\left(T_{0}, z \frac{z}{h^{*}} T_{1}\right) d z\left(\bar{N}_{y}, \bar{M}_{y}\right)=\int_{\frac{-h^{*}}{2}}^{\frac{h^{*}}{2}}\left(\bar{Q}_{12} \alpha_{x}+\bar{Q}_{22} \alpha_{y}+\bar{Q}_{26} \alpha_{x y}\right)\left(T_{0}, z \frac{z}{h^{*}} T_{1}\right) d z \\
\left(\bar{N}_{x y}, \bar{M}_{x y}\right)=\int_{\frac{-h^{*}}{2}}^{\frac{h^{*}}{2}}\left(\bar{Q}_{16} \alpha_{x}+\bar{Q}_{26} \alpha_{y}+\bar{Q}_{66} \alpha_{x y}\right)\left(T_{0}, z \frac{z}{h^{*}} T_{1}\right) d z\left(\tilde{N}_{x}, \tilde{M}_{x}\right)=\int_{\frac{-h^{*}}{2}}^{\frac{h^{*}}{2}} \tilde{e}_{31} H_{z}\left(1, z^{2}\right) d z \quad\left(\tilde{N}_{y}, \tilde{M}_{y}\right)=\int_{\frac{h^{*}}{2}}^{\frac{h^{*}}{2}} \tilde{e}_{32} H_{z}\left(1, z^{2}\right) d z \\
\left(\tilde{N}_{x y}, \tilde{M}_{x y}\right)=\int_{\frac{-k^{*}}{2}}^{\frac{h^{*}}{2}} \tilde{e}_{36} H_{z}\left(1, z^{2}\right) d z \quad\left(A_{i j}, B_{i j}, D_{i j}\right)=\int_{\frac{-h^{*}}{2}}^{\frac{h^{*}}{2}} \bar{Q}_{i j}\left(1, z, z^{2}\right) d z(i, j=1,2,6) \\
(\rho, H)=\int_{\frac{-h^{*}}{2}}^{\frac{h^{*}}{2}} \rho_{0}(1, z) d z
\end{gathered}
$$

in which $\rho_{0}$ is the density of ply, $p_{1}$ and $p_{2}$ are the in-plane distributed forces, $q$ is the applied pressure load.

\subsection{Dynamic Discretized Equations}

Without the shear deformation effect, we apply the weighting coefficients of discretized equations (2) in the two-dimensional generalized differential qradrature (GDQ) method to discrete the differential equations (4) under the vibration of time sinusoidal displacement and temperature:

$$
\begin{gathered}
u=\left[u^{0}(x, y)\right] \sin \left(\omega_{m n} t\right), v=\left[v^{0}(x, y)\right] \sin \left(\omega_{m n} t\right), \\
w=w(x, y) \sin \left(\omega_{m n} t\right), \Delta T=\left[T_{0}(x, y)+\frac{z}{h^{*}} T_{1}(x, y)\right] \sin (\gamma t) .
\end{gathered}
$$

of applied heat flux.

And the following non-dimensional parameters are introduced:

$$
\begin{gathered}
X=x / a, \quad Y=y / b, U=u^{0} / a, V=v^{0} / b \\
W=10 h^{*} w /\left(\alpha_{x} \bar{T}_{1} a^{2}\right)
\end{gathered}
$$

under the vibration of time sinusoidal displacement and temperature.

We obtain the following dynamic discretized equations in matrix notation:

$$
[A M]\{S U V W\}+[K E]\{S W\}+[F Q]\{U V W\}=\{F\}
$$

where

where $\omega_{m n}$ is natural frequency of the plate, $\gamma$ is frequency

$$
\begin{aligned}
& \{S U V W\}=\left\{\sum_{l=1}^{N} A_{i, l}^{(2)} U_{l, j} \quad \sum_{l=1}^{N} A_{i, l}^{(1)} \sum_{m=1}^{M} B_{j, m}^{(1)} U_{l, m} \sum_{m=1}^{M} B_{j, m}^{(2)} U_{i, m} \quad \sum_{l=1}^{N} A_{i, l}^{(2)} V_{l, j}\right. \\
& \left.\sum_{l=1}^{N} A_{i, l}^{(1)} \sum_{m=1}^{M} B_{j, m}^{(1)} V_{l, m} \quad \sum_{m=1}^{M} B_{j, m}^{(2)} V_{i, m} \quad \sum_{l=1}^{N} A_{i, l}^{(2)} W_{l, j} \quad \sum_{l=1}^{N} A_{i, l}^{(1)} \sum_{m=1}^{M} B_{j, m}^{(1)} W_{l, m} \quad \sum_{m=1}^{M} B_{j, m}^{(2)} W_{i, m}\right\}^{t} \quad\{S W\}=\left\{\sum_{l=1}^{N} A_{i, l}^{(1)} W_{l, j} \quad \sum_{m=1}^{M} B_{j, m}^{(1)} W_{i, m}\right\}^{t} \\
& \{U V W\}=\left\{U_{i, j} \quad V_{i, j} \quad W_{i, j}\right\}^{t} \\
& \{F\}=\left\{\begin{array}{lll}
F_{1} & F_{2} & F_{3}
\end{array}\right\}^{t}
\end{aligned}
$$

The elements of $3 \times 9$ matrix $[A M], 3 \times 2$ matrix $[K E]$ and $3 \times 3$ matrix $[F Q]$ are as follows:

$$
\begin{gathered}
A M_{11}=\left(A_{11} / a\right) \sin \left(\omega_{m n} t\right) \quad A M_{12}=\left(2 A_{16} / b\right) \sin \left(\omega_{m n} t\right) \quad A M_{13}=\left(A_{66} a / b^{2}\right) \sin \left(\omega_{m n} t\right) \\
A M_{14}=\left(A_{16} b / a^{2}\right) \sin \left(\omega_{m n} t\right) \quad A M_{15}=\left[\left(A_{12}+A_{66}\right) / a\right] \sin \left(\omega_{m n} t\right)
\end{gathered}
$$




$$
\begin{aligned}
& A M_{16}=\left(A_{26} / b\right) \sin \left(\omega_{m n} t\right) \quad A M_{17}=A M_{18}=A M_{19}=0 \quad A M_{21}=\left(A_{16} / a\right) \sin \left(\omega_{m n} t\right) \quad A M_{22}=\left[\left(A_{12}+A_{66}\right) / b\right] \sin \left(\omega_{m n} t\right) \\
& A M_{23}=\left(A_{26} a / b^{2}\right) \sin \left(\omega_{m n} t\right) \\
& A M_{24}=\left(A_{66} b / a^{2}\right) \sin \left(\omega_{m n} t\right) \quad A M_{25}=\left(2 A_{26} / a\right) \sin \left(\omega_{m n} t\right) \\
& A M_{26}=\left(A_{22} / b\right) \sin \left(\omega_{m n} t\right) \quad A M_{27}=A M_{28}=A M_{29}=0 \\
& A M_{31}=\left[\left(B_{11}+B_{16}\right) / a\right] \sin \left(\omega_{m n} t\right) \\
& A M_{32}=\left[\left(2 B_{16}+B_{12}+B_{66}\right) / b\right] \sin \left(\omega_{m n} t\right) \\
& A M_{33}=\left[\left(B_{66}+B_{26}\right) a / b^{2}\right] \sin \left(\omega_{m n} t\right) \\
& A M_{34}=\left[\left(B_{16}+B_{66}\right) b / a^{2}\right] \sin \left(\omega_{m n} t\right) \\
& A M_{35}=\left(B_{12}+B_{66}+2 B_{26}\right)(1 / a) \sin \left(\omega_{m n} t\right) A M_{36}=\left[\left(B_{26}+B_{22}\right) / b\right] \sin \left(\omega_{m n} t\right) \\
& A M_{37}=0 \\
& A M_{38}=0 \\
& A M_{39}=0 \\
& K E_{11}=-\frac{\alpha_{x} \bar{T}_{1} a^{2}}{10 h^{*}}\left[\frac{1}{a} k_{c} c(t) \sum_{k=1}^{N_{k}} \tilde{e}_{31}\left(z_{k}-z_{k-1}\right)\right] \omega_{m n} \cos \left(\omega_{m n} t\right) K E_{12}=-\frac{\alpha_{x} \bar{T}_{1} a^{2}}{10 h^{*}}\left[\frac{1}{b} k_{c} c(t) \sum_{k=1}^{N_{k}} \tilde{e}_{36}\left(z_{k}-z_{k-1}\right)\right] \omega_{m n} \cos \left(\omega_{m n} t\right) \\
& K E_{21}=-\frac{\alpha_{x} \bar{T}_{1} a^{2}}{10 h^{*}}\left[\frac{1}{a} k_{c} c(t) \sum_{k=1}^{N_{k}} \tilde{e}_{36}\left(z_{k}-z_{k-1}\right)\right] \omega_{m n} \cos \left(\omega_{m n} t\right) K E_{22}=-\frac{\alpha_{x} \bar{T}_{1} a^{2}}{10 h^{*}}\left[\frac{1}{b} k_{c} c(t) \sum_{k=1}^{N_{k}} \tilde{e}_{32}\left(z_{k}-z_{k-1}\right)\right] \omega_{m n} \cos \left(\omega_{m n} t\right) \\
& K E_{31}=K E_{32}=0 \\
& F Q_{11}=\rho \omega_{m n}^{2} a \sin \left(\omega_{m n} t\right) \quad F Q_{12}=F Q_{13}=0 \\
& F Q_{22}=\rho \omega_{m n}^{2} b \sin \left(\omega_{m n} t\right) \quad F Q_{21}=F Q_{23}=0 \\
& F Q_{31}=F Q_{32}=0 \quad F Q_{33}=-\rho \omega_{m n}^{2}\left[\alpha_{x} \bar{T}_{1} a^{2} /\left(10 h^{*}\right)\right] \sin \left(\omega_{m n} t\right)
\end{aligned}
$$

in which $F_{1}, F_{2}, F_{3}$ are represented in the following discretized equation:

$$
\begin{aligned}
F_{1}= & \left(\frac{1}{a} \sum_{l=1}^{N} A_{i, l}^{(1)} \bar{N}_{x_{l, j}}+\frac{1}{b} \sum_{m=1}^{M} B_{j, m}^{(1)} \bar{N}_{x y_{i, m}}\right) \sin (\gamma t)+p_{1_{i, j}} \\
F_{2}= & \left(\frac{1}{a} \sum_{l=1}^{N} A_{i, l}^{(1)} \bar{N}_{x y_{l, j}}+\frac{1}{b} \sum_{m=1}^{M} B_{j, m}^{(1)} \bar{N}_{y_{i, m}}+p_{z_{i, j}}\right) \sin (\gamma t) \\
F_{3}= & q_{i, j}+\left(\frac{1}{a} \sum_{l=1}^{N} A_{i, l}^{(1)} \bar{M}_{x_{l, j}}+\frac{1}{b} \sum_{m=1}^{M} B_{j, m}^{(1)} \bar{M}_{x y_{i, m}}\right) \sin (\gamma t) \\
& +\left(\frac{1}{a} \sum_{l=1}^{N} A_{i, l}^{(1)} \bar{M}_{x y_{l, j}}+\frac{1}{b} \sum_{m=1}^{M} B_{j, m}^{(1)} \bar{M}_{y_{l, m}}\right) \sin (\gamma t)
\end{aligned}
$$

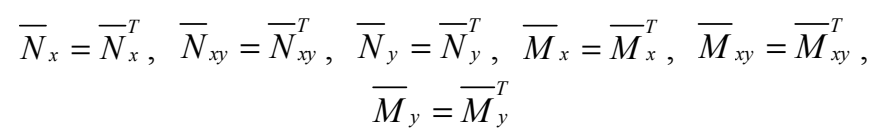

in which $\left\{\bar{N}^{T}\right\}$ is the thermal force resultant, $\left\{\bar{M}^{T}\right\}$ is the thermal moment resultant.

\section{Some Numerical Results and Discussions}

The typical upper surface magnetostrictive layer of the three-layer $\left(0^{\circ} \mathrm{m} / 90^{\circ} / 0^{\circ}\right)$ cross-ply laminates plate under four sides simply supported are considered, the superscript of in which $p_{1}$ and $p_{2}$ are the in-plane distributed forces, $q$ is the applied pressure load. The force resultants $\bar{N}_{x}, \bar{N}_{x y}, \bar{N}_{y}$ and moment resultants $\bar{M}_{x}, \bar{M}_{x y}, \bar{M}_{y}$ are expressed as follows: $m$ denotes magnetostrictive layer. The elastic modules, material conductivity and specific heat of the typical host material and Terfenol-D magnetostrictive material are used the same value as in [10]. The grid points are used in the following coordinates: 


$$
\begin{aligned}
& x_{i}=0.5\left[1-\cos \left(\frac{i-1}{N-1} \pi\right)\right] a, i=1,2, \ldots, N \\
& y_{j}=0.5\left[1-\cos \left(\frac{j-1}{M-1} \pi\right)\right] b, j=1,2, \ldots, M
\end{aligned}
$$

Firstly, we make the dynamic convergence studies of center deflection amplitude $W(a / 2, b / 2)$ without shear effects in the thermal vibration of sinusoidal temperature only $\left(T_{0}=0\right.$, $\left.\bar{T}_{1}=1.0^{\circ} \mathrm{F}, p_{1}=p_{2}=q=0\right)$ at time 6sec, $m=n=1$ mode shape, with $k_{c} c(t)=10^{8}$, aspect ratio $a / b=1.0$, side-to-thickness ratio $a / h^{*}=100,50,20$ and 10. Figure 1 shows that $W(a / 2, b / 2)$ in the grid point $N \times M=9 \times 9$, $11 \times 11$ and $13 \times 13$ of GDQ method for the three-layer $\left(0^{\circ} \mathrm{m} / 90^{\circ} / 0^{\circ}\right)$ laminated plate. The number of grid points in

$N \times M=13 \times 13$ are found for $W(a / 2, b / 2)$ in the convergence result and use further in the GDQ analyses of time responses for deflection and stress for $a / h^{*}=100,50,20$ and 10. Figure 2 shows that deflection amplitude $W(X, b / 2)$ in the grid point $N \times M=13 \times 13$ of GDQ method for the thick $\left(0^{\circ} \mathrm{m} / 90^{\circ} / 0^{\circ}\right)$ laminated plate $a / h^{*}=10$ without shear effects. The maximum value of deflection amplitude (0.00016) occur nearly at the center position $(x=a / 2, y=b / 2)$.

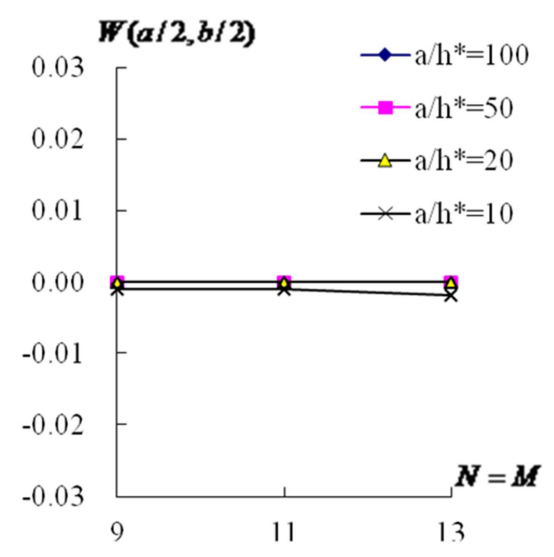

Figure 1. Convergence for $\left(0^{\circ} \mathrm{m} / 90^{\circ} / 0^{\circ}\right)$.

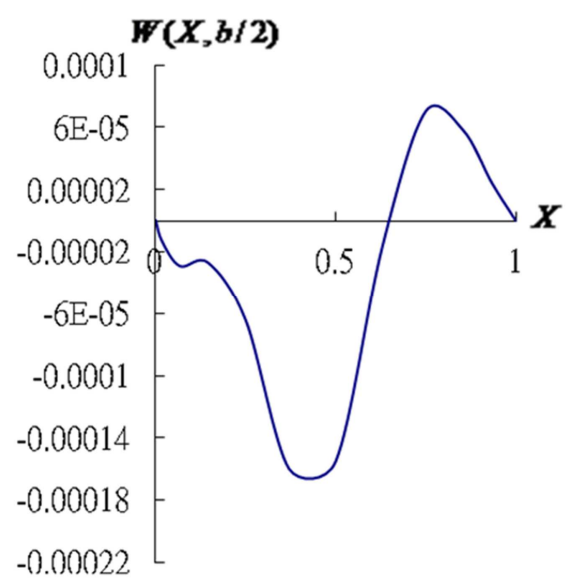

Figure 2. $W(X, b / 2)$ vs. $X$ for $a / h^{*}=10$.
The same control gain $k_{c} c(t)$ values are used as in analysis of Hong [10] to calculate the displacement and stress of typical three-layer $\left(0^{\circ} \mathrm{m} / 90^{\circ} / 0^{\circ}\right)$ laminated magnetostrictive plate without shear effects. Figure 3 and Figure 4 show that the time response of the non-dimensional transverse center deflection amplitude $W(a / 2, b / 2)$ with respective to time for thick $a / h^{*}=10$ and thin $a / h^{*}=100$ laminated plate, respectively. And the controlled values of displacement $W(a / 2, b / 2)$ without/with shear effects are compared. The $W(a / 2, b / 2)$ without shear effect are found in smaller value than the $W(a / 2, b / 2)$ with shear effect by using the GDQ computation method, typically in the thinner plate $\left(a / h^{*}=100\right)$.
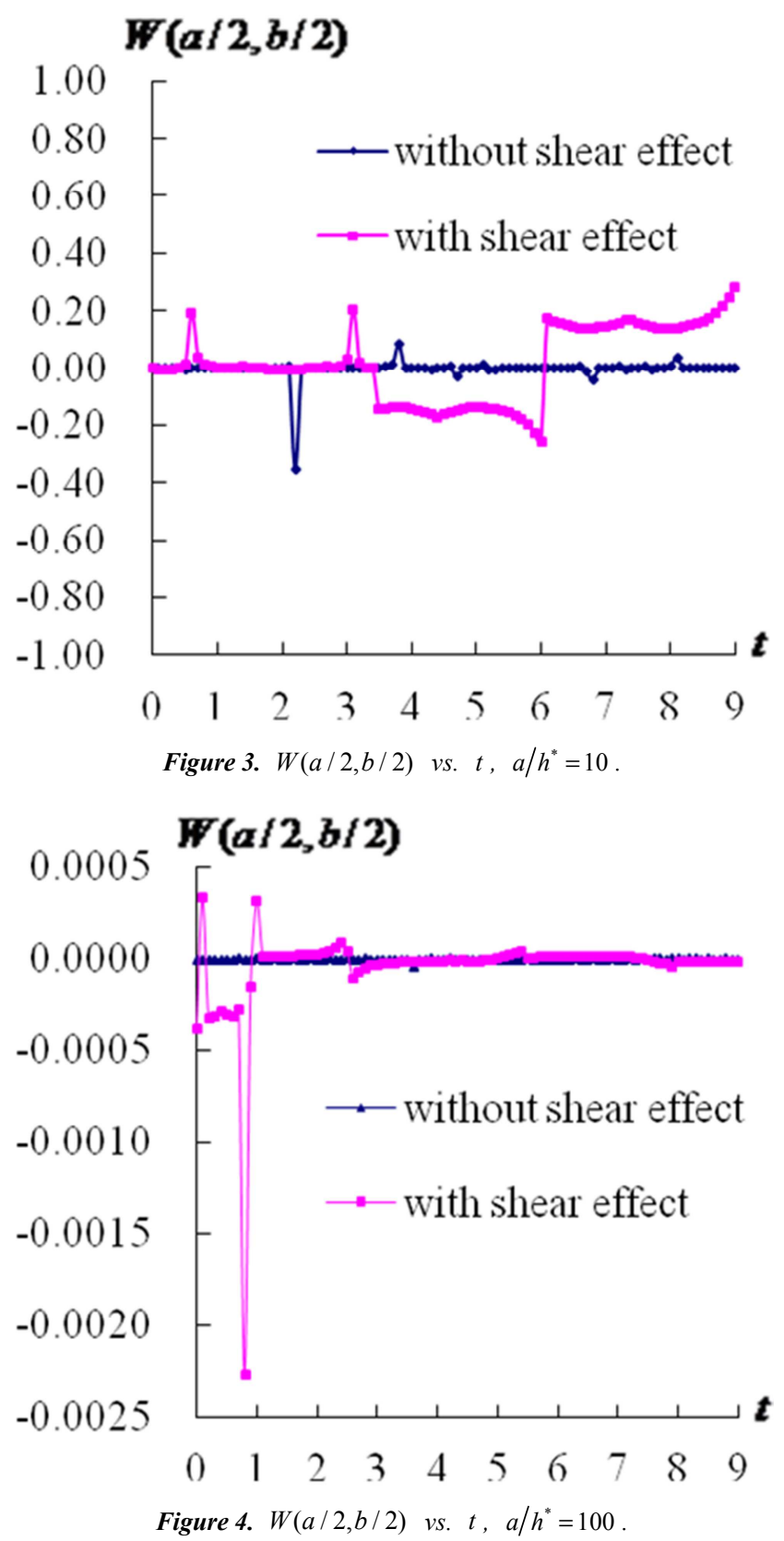

Figure 5 and Figure 6 show that the time response of the 
dominated non-dimensional stress $\bar{\sigma}_{x}=\sigma_{x} h^{*} /\left(\alpha_{x} \bar{T}_{1} a E_{2}\right)$ at center position of lower surface $Z=-0.5 h^{*}$ with respective to time for thick $a / h^{*}=10$ and thin $a / h^{*}=100$ laminated plate, respectively. And the controlled values of stress $\bar{\sigma}_{x}$ without/with shear effects are compared. The $\bar{\sigma}_{x}$ without shear effect are keep constant and found in smaller value than the $\bar{\sigma}_{x}$ with shear effect by using the GDQ computation method, typically in the thinner plate $\left(a / h^{*}=100\right)$.

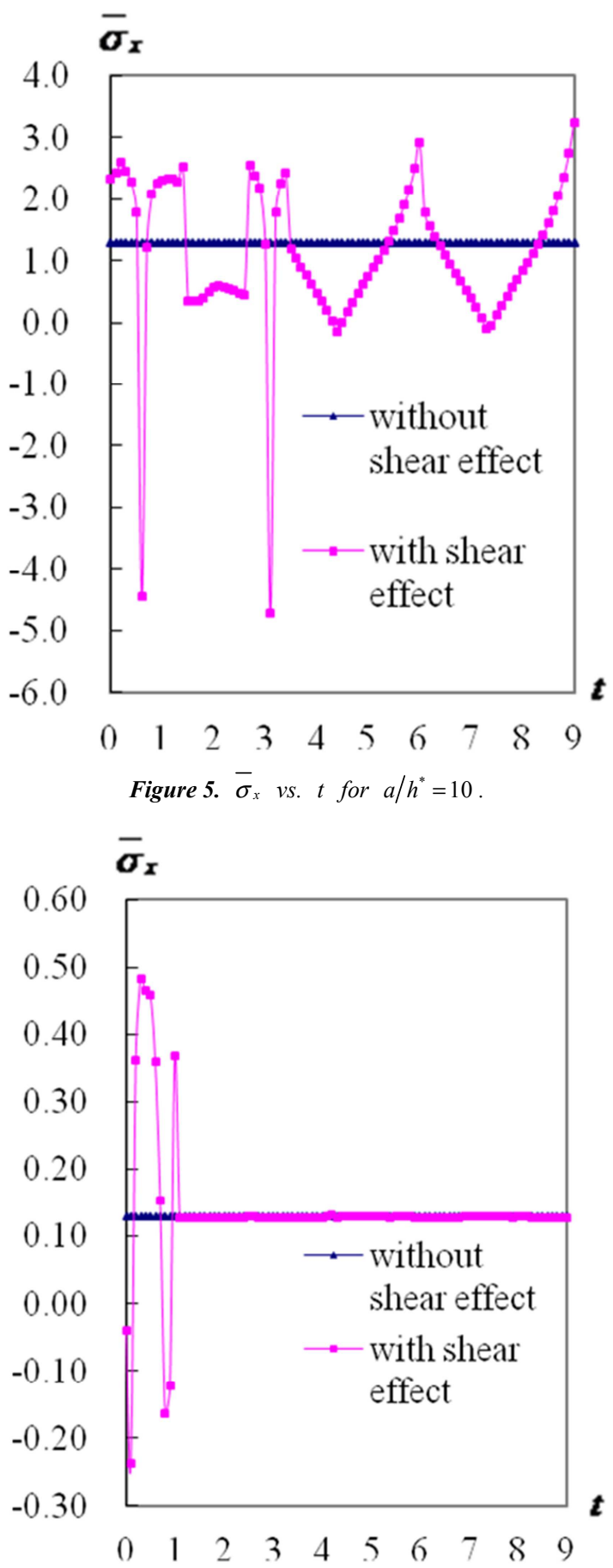

Figure 6. $\bar{\sigma}_{x}$ vs. $t$ for $a / h^{*}=100$.

\section{Conclusions}

The GDQ provides a method to compute the controlled deflection and stress in the cross $\left(0^{\circ} \mathrm{m} / 90^{\circ} / 0^{\circ}\right)$ ply laminated magnetostrictive plate subjected to thermal vibration of sinusoidal temperature without shear deformation effect. Without/with shear deformation effect, especially, in the thin plate $a / h^{*}=100$, the amplitude of transverse center deflection $W(a / 2, b / 2)$ can be controlled into a smaller desirable value with the suitable control gain $k_{c} c(t)$ value, by using the GDQ method. Without shear deformation effect, the amplitude of the dominated stress $\bar{\sigma}_{x}$ is the same value under the corresponding controlled $W(a / 2, b / 2)$ condition in the thick plate $a / h^{*}=10$ and thin plate $a / h^{*}=100$ of the GDQ method. In the moderately thick plate $a / h^{*}=10$ of laminated magnetostrictive plate, the effect of shear deformation should be considered for the computational controlled values of transverse center deflection $W(a / 2, b / 2)$ and dominated stress $\bar{\sigma}_{x}$. In the future work, parametric study would be investigated by using the nonlinear coefficient term in the third-order shear deformation theory (TSDT) of displacement fields to calculate the displacement and stress for the thick plates.

\section{References}

[1] Arani A. G., Maraghi Z. K., A feedback control system for vibration of magnetostrictive plate subjected to follower force using sinusoidal shear deformation theory. Ain Shams Engineering Journal 2016; 7: 361-369.

[2] Zhang Y., Zhou H., Zhou Y., Vibration suppression of cantilever laminated composite plate with nonlinear giant magnetostrictive material layers. Acta Mechanica Solida Sinica 2015; 28: 50-61.

[3] Sarangan S., Singh B. N., Higher-order closed-form solution for the analysis of laminated composite and sandwich plates based on new shear deformation theories. Composite Structures 2016; 138: 391-403.

[4] Hong CC., Rapid heating induced vibration of magnetostrictive functionally graded material plates. Transactions of the ASME, Journal of Vibration and Acoustics 2012; 134: 021019, pp.1-11.

[5] Nguyen T. K., Sab K., Bonnet G., First-order shear deformation plate models for functionally graded materials. Composite Struct 2008; 83: 25-36.

[6] Ramirez F., Heyliger P. R., Pan E., Free vibration response of two-dimensional magneto-electro-elastic laminated plates. J of Sound and Vibration 2006; 292: 626-644.

[7] Lee S. J., Reddy J. N., Non-linear response of laminated composite plates under thermomechanical loading. Int $\mathrm{J}$ of Non-linear Mech 2005; 40: 971-985.

[8] Lee S. J., Reddy J. N., Rostam-Abadi F., Transient analysis of laminated composite plates with embedded smart-material layers. Fin Elem in Analysis and Design 2004; 40: 463-483. 
[9] Hong C. C., Thermal vibration and transient response of magnetostrictive functionally graded material plates. European Journal of Mechanics A/Solids 2014; 43: 78-88.

[10] Hong C. C., Transient responses of magnetostrictive plates without shear effects. International Journal of Engineering Science 2009; 47: 355-362.

[11] Bert C. W., Jang S. K., Striz A. G., Nonlinear bending analysis of orthotropic rectangular plates by the method of differential quadrature. Comput Mech 1989; 5: 217-226.
[12] Shu C., Du H., Implementation of clamped and simply supported boundary conditions in the GDQ free vibration analyses of beams and plates. Int J of Solds and Struct 1997; 34: 819-835.

[13] Whitney J. M., Structural analysis of laminated anisotropic plates. Lancaster, PA, USA: Technomic Publishing Company, Inc., 1987. 